\title{
Indução à Ovulação pelo Uso de LHRH Análogo e Fertilização Artificial em Rã-Touro (Rana catesbeiana $)^{1}$
}

\section{Claudio Angelo Agostinho², Francisco Stefano Wechsler², Paulo Eduardo de Oliveira Nictheroy ${ }^{3}$, Daniela Felipe Pinheiro 4}

\begin{abstract}
RESUMO - Este trabalho teve por objetivo aperfeiçoar a técnica de reprodução induzida existente para rã-touro, com o intuito de aumentar a taxa de fecundidade e viabilizar seu uso pelo produtor. As doses hormonais para a indução da ovulação e espermiação seguiram as propostas de FALCON e CULLEY (1995) e ALONSO (1997); entretanto, a técnica de fertilização artificial foi adaptada da metodologia para reprodução artificial de peixes com ovos não-aderentes (WOYNAROVICH e HORVÁTH, 1983). A técnica proposta apresenta as seguintes etapas: I) sincronização da ovulação e da espermiação, por meio de hormônio liberador de gonadotropina ((Des-Gli ${ }^{10}$, DHis(Bzl) ${ }^{6}$, Pro-NHEt $\left.{ }^{9}\right)$-LHRH)); II) extração dos óvulos de cada fêmea (1 a 2 minutos); III) fertilização dos óvulos (2 minutos) com líquido espermático diluído em $100 \mathrm{~mL}$ de água; IV) hidratação dos ovos em 10 a 20 litros de água; e V) incubação dos ovos em quadros de tela de 1x 0,70 m, com malha de $1 \mathrm{~mm}$. As taxas de fertilização obtidas com as modificações propostas foram superiores a $60 \%$. Ressalta-se ainda que a técnica propiciou a obtenção, a partir de um mesmo animal, de várias desovas, sendo que cada fêmea pode ovular em intervalos de, aproximadamente, 45 dias.
\end{abstract}

Palavras-chave: fertilização artificial, LHRHa, Rana catesbeiana, reprodução

\section{Spawning Inducing by Analog LHRH and Artificial Fertilization of Bullfrog (Rana catesbeiana)}

\begin{abstract}
The objective of this study was to improve the artificial fertilization techniques for bullfrog, to increase the fertilization rate and become their use accessible for frog producers. Hormone dosages for inducing ovulation and spermiation followed those proposed by FALCON and CULLEY (1995) and ALONSO (1997); however, the artificial fertilization technique was adapted from the available methodology for artificial reproduction of fish with non-adhering eggs (WOYNAROVICH e HORVÁTH, 1983). The proposed technique consists of the following steps: I) synchronization of ovulation and spermiation through gonadotropin releasing hormone ((Des-Gli ${ }^{10}$, D-His(Bzl $)^{6}$, Pro-NHEt $\left.\left.{ }^{9}\right)-\mathrm{LHRH}\right)$ ); II) complete ovule extrusion in every female (1 to 2 minutes); III) ovule fertilization (2 minutes) with sperm diluted in $100 \mathrm{~mL}$ water; IV) ova hydration in 10 - $20 \mathrm{~L}$ water; V) ova incubation in screen frames, measuring 1,0 x 0,7 m, with $1 \mathrm{~mm}$ screen size. Fertilization rate obtained with the proposed modifications stayed above $60 \%$. It should also be emphasized that the proposed techniques allowed to obtain several spawns from the same frog, and each female was able to ovulate, approximately, every 45 days.
\end{abstract}

Key Words: artificial fertilization, bullfrog, LHRH, reproduction

\section{Introdução}

A reprodução de rãs na maioria dos ranários comerciais é cíclica, ocorrendo picos de desovas no verão e somente algumas na primavera, no outono e inverno. Esta sazonalidade da reprodução compromete o cronograma de produção dos ranários, pois a produção de girinos e imagos fica restrita a alguns meses do ano; conseqüentemente, os galpões de recria e os tanques de girinos permanecem vazios durante vários meses.
As fêmeas adultas de rã-touro permanecem maduras durante o ano todo (COSTA, 1992), todavia, o processo de maturação final e ovulação somente é desencadeado por estímulos ambientais, como as chuvas e o aumento da temperatura logo após o final do inverno. As técnicas de indução à ovulação e fertilização artificial têm importante papel na resolução deste problema, pois usam hormônios liberadores de gonadotropina para desencadear o processo reprodutivo, tornando desnecessários os estímulos ambientais e possibilitando a sincronização das desovas

\footnotetext{
${ }^{1}$ Projeto Financiado pela FAPESP (processo ㄲo $96 / 03405-4$ ).

2 Professor Assistente Dr., DPEA, FMVZ, UNESP - Botucatu-SP. E-mail: agostinho@fca.unesp.br

3 Zootecnista. DPEA, FMVZ, UNESP - Botucatu, SP.

${ }^{4}$ Bolsista Fapesp - DPEA, FMVZ, UNESP - Botucatu, SP.
} 
para o período desejado.

Os primeiros trabalhos de indução à reprodução de rã-touro pelo uso de hipófises foram realizados por RUGH (1935). Em 1974, o NATIONAL ACADEMY OF SCIENCE - NAS propôs a utilização de duas hipófises misturadas com $5 \mathrm{mg}$ de progesterona para a indução da ovulação. $O$ trabalho mais recente de indução à reprodução de rã-touro é o de RIBEIRO FILHO (1998a), que trabalhou com várias dosagens de hipófise e obteve os melhores resultados com 5 ou $7 \mathrm{mg} / \mathrm{kg}$ aplicados em duas doses (10\% do total na primeira e o restante na segunda dose, 12 horas após).

A indução de desovas pelo uso de hipófises não tem sido empregada por produtores de rãs, principalmente pela dificuldade de extração da glândula, que só pode ser feita por um técnico que tenha bom conhecimento de anatomia. Além disso, pode ocorrer variação na quantidade de gonadotropina, dependendo do estádio de maturação gonadal da fêmea doadora da hipófise.

Uma solução viável para o produtor será o uso de hormônios liberadores de gonadotropina análogos, que têm apresentado excelentes resultados na indução da ovulação e espermiação, são facilmente adquiridos e gozam de fácil dosagem e aplicação. Entre eles pode-se citar o acetato de buserelina (ALONSO, 1997), com o nome comercial de Conceptal e fabricado pela Hoescht do Brasil, e o análogo ((Des-Gli ${ }^{10}$, D-His $(\mathrm{Bzl})^{6}$, Pro-NHEt ${ }^{9}$ )-LHRH)) (FALCON e CULLEY, 1995), fabricado pela BACHEM California-USA.

O emprego de hormônio sintético para a indução a ovulação e espermiação é uma técnica rotineira em laboratórios. Entretanto, a utilização da técnica de fertilização artificial em ranários comerciais ainda é limitada, devido à sua complexidade e à baixa fecundidade obtida, quando se emprega a metodologia descrita nos trabalhos publicados até o momento.

O objetivo deste trabalho foi propor modificações nas técnicas de fertilização artificial de rãs, com o intuito de aumentar a taxa de fecundidade e viabilizar seu emprego pelo ranicultor e nos trabalhos de melhoramento genético.

\section{Material e Métodos}

\section{Descrição da técnica de fertilização artificial}

As doses hormonais empregadas na indução da ovulação e espermiação seguiram as propostas de FALCON e CULLEY (1995) e ALONSO (1997). Entretanto, a técnica de fertilização artificial foi adaptada da metodologia para reprodução artificial de peixes com ovos não-aderentes (WOYNAROVICH e HORVÁTH, 1983) e será descrita nos parágrafos subseqüientes:

a) Indução à ovulação. Aplicação de $10 \mu \mathrm{g}$ de hormônio liberador de gonadotropina ((Des-Gli ${ }^{10}, \mathrm{D}-$ His(Bzl ${ }^{6}$, Pro-NHEt $\left.{ }^{9}\right)$-LHRH)) por kg de peso vivo, a cada 12 horas, até a ovulação, que ocorre de 24 a 36 horas após a primeira aplicação.

b) Coleta de esperma. Aplicação de $10 \mu \mathrm{g}$ de LHRHa por $\mathrm{kg}$ de peso vivo. Uma hora após a aplicação, o esperma é retirado, introduzindo a ponta de uma pipeta de 2,0 $\mathrm{mL}$ na cloaca do macho e coletando de 2,0 a 4,0 mL de esperma (Figura 1). São usados dois a três machos para cada fêmea, e a mistura de esperma deve ser diluída em $100 \mathrm{~mL}$ de água, pouco antes da fertilização artificial.

c) Extração dos óvulos. A extração é realizada segurando a fêmea pelas patas posteriores e comprimindo levemente o ventre com o auxílio do polegar. Os óvulos são coletados em recipiente seco e limpo. O tempo necessário para o completo esgotamento do ovário é de, aproximadamente, um minuto (Figura 2).

d) Fertilização artificial. O esperma diluído é vertido sobre os óvulos, agitando-se a mistura por, aproximadamente, dois minutos. Os ovos fecundados são misturados em 15 litros de água, até a completa hidratação. Após a hidratação, os ovos são colocados em incubadoras de madeira flutuantes providas de fundode tela plástica com malhade $1,0 \mathrm{~mm}(1,0 \mathrm{mx} 0,70 \mathrm{~m})$, de acordo com as recomendações de LIMA e AGOSTINHO (1988).

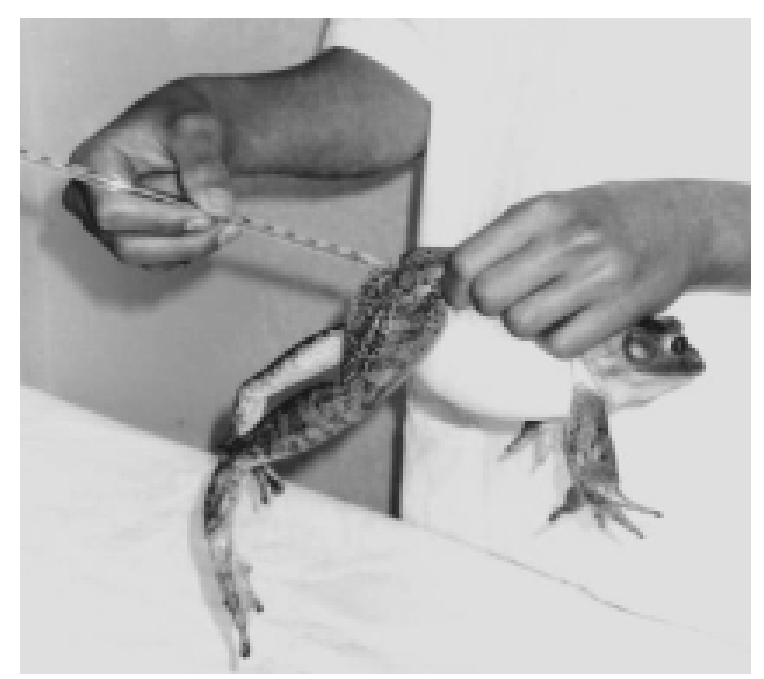

Figura 1 - Retirada de esperma de rã-touro utilizando uma pipeta.

Figure 1 - Extracting sperm from bullfrog with a pipette. 
Rev. bras. zootec.

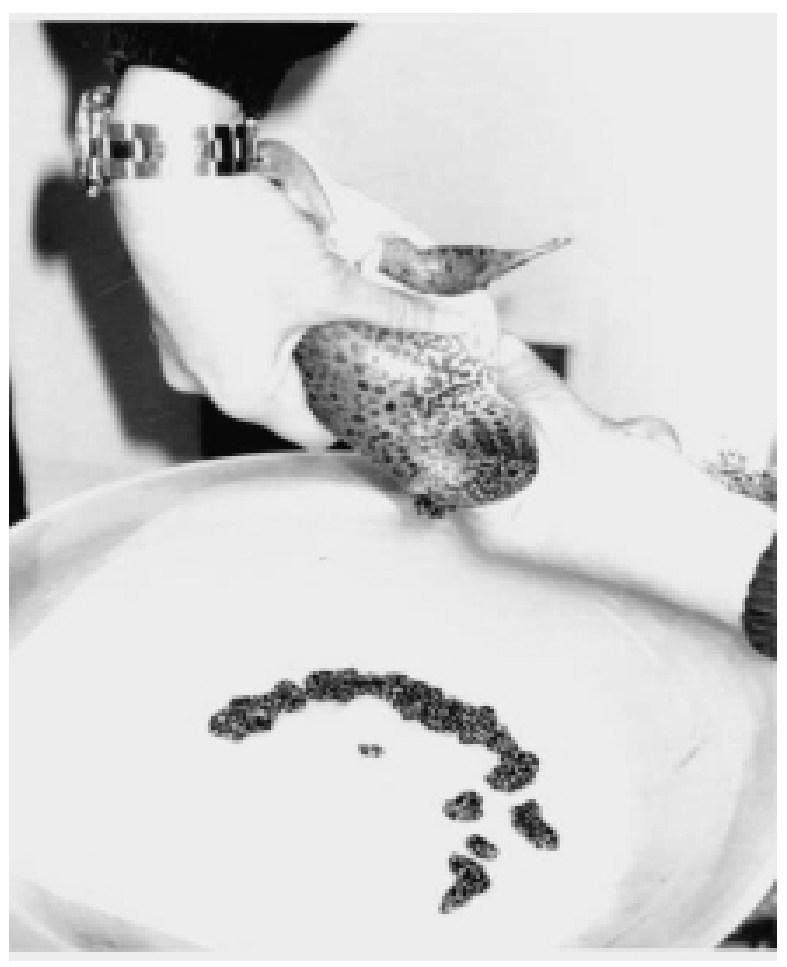

Figura 2 - Retirada dos óvulos pela compressão do ventre de rã-touro.

Figure 2 - Extracting eggs from a female bullfrog.

\section{Avaliação da técnica de fertilização artificial}

Fêmeas e machos de primeira maturação foram adquiridos no mês de abril de 1998, de um ranário comercial. Os animais foram marcados individualmente, segundo a codificação proposta por MARTOFF (1953), e colocados em baias aquecidas, com temperatura do ar variando de 24 a $28^{\circ} \mathrm{C}$ e fotoperíodo controlado para 12 horas de luz.

A alimentação foi feita diariamente à vontade, por meio de cochos vibratórios, com ração comercial extrusada Rana-45 (Nutremix Premix e Rações Ltda.), que continha $45 \%$ de proteína bruta, $2160 \mathrm{kcal} / \mathrm{kg}$ de energia metabolizável, 4\% de extrato etéreo, $6 \%$ de fibra bruta, $2,5 \%$ de cálcio e $1,4 \%$ de fósforo.

Indução à ovulação

Vinte e três fêmeas foram avaliadas quanto à produção de óvulos e à resposta à indução da ovulação. As fêmeas foram induzidas em três períodos: 21 a 30 de julho de 1998; 5 a 18 de setembro de 1998; e 14 a 27 de outubro de 1998.

Os óvulos extraídos foram pesados e o número total de óvulos, estimado por contagem de amostras com, aproximadamente, $1 \mathrm{~g}$.

Viabilidade dos óvulos e do esperma

A viabilidade dos óvulos e do esperma foi avaliada nas desovas do período de 20 a 31 de julho de 1998 . Cada desova foi dividida em cinco amostras, com, aproximadamente, 4,0 g de ovos. Cada amostra foi fecundada por um macho diferente e a fecundidade, estimada com base na taxa de eclosão.

Avaliação da técnica de fertilização artificial na produção massal de girinos

Esta avaliação foi feita com o intuito de verificar a viabilidade do uso desta técnica pelo produtor. Nesse caso, as desovas foram realizadas simulando uma situação de campo, em que o produtor não possui microscópio para verificar a viabilidade e concentração de espermatozóides. Cada fêmea foi fecundada com o esperma de três reprodutores, com o intuito de contrabalançar possível baixa fertilidade de algum macho. A avaliação ocorreu no período de 14 a 27 de outubro de 1998, em 13 fêmeas. Após a fecundação, os ovos foram colocados em incubadoras de madeira flutuantes com fundo de tela plástica e malha de 1,0 mm (1,0 m x 0,70 m). Após a eclosão, avaliou-se a taxa de fecundação.

\section{Metodologia estatística}

As proporções de fêmeas que desovaram em julho, setembro e outubro foram analisadas, quanto às diferenças entre períodos, por meio do teste do quiquadrado. Os dados de peso das fêmeas, peso dos óvulos, número de ovos (transformado logaritmicamente) e índice ovócito somático (IOS) foram submetidos à análise de variância, pelo programa GLM (SAS, 1986), mediante o modelo 1:

$$
\mathrm{Y}_{\mathrm{ij}}=\mathrm{m}+\mathrm{F}_{\mathrm{i}}+\mathrm{P}_{\mathrm{j}}+\mathrm{e}_{\mathrm{ij}}
$$

em que $\mathrm{Y}_{\mathrm{ij}}$ é variável observada na i-ésima fêmea e j-ésimo período; $m$, média da população; $F_{i}$, efeito aleatório de fêmea, NID $\left(0, \sigma_{F}^{2}\right) ; P_{j}$, efeito fixo de período; $\mathrm{e}_{\mathrm{ij}}$,erro aleatório, $\operatorname{NID}\left(0, \sigma_{\mathrm{e}}^{2}\right)$.

As médias de quadrados mínimos de períodos foram comparadas por meio do teste Tukey-Kramer (KRAMER, 1957).

A porcentagem de fecundação (submetida à transformação angular), obtida na desova de julho, foi analisada por intermédio do modelo 2 :

$$
\mathrm{Y}_{\mathrm{ij}}=\mathrm{m}+\mathrm{F}_{\mathrm{i}}+\mathrm{e}_{\mathrm{ij}}
$$

em que $Y_{i j}$ é porcentagem de fecundação, obtida com a i-ésima fêmea e j-ésimo macho; e demais efeitos como no modelo 1. 
Como não foi possível fixar intervalo constante da verificação da ovulação à extração dos óvulos, optou-se, como alternativa ao modelo 1, por incluir o tempo decorrido entre estes dois eventos, como covariável associada à fêmea, o que levou ao modelo 3 :

$$
\mathrm{Y}_{\mathrm{ij}}=\mathrm{m}+\mathrm{F}_{\mathrm{i}}+\beta\left(\mathrm{t}_{\mathrm{i}}-\overline{\mathrm{t}} .\right)+\mathrm{e}_{\mathrm{ij}}
$$

em que $b$ é o coeficiente de regressão; $t_{i}$, tempo decorrido em minutos decorridos após a constatação da ovulação até a extração dos óvulos; e os demais efeitos como no modelo 2 .

Ao modelo 3 adicionou-se o número de espermatozóides como covariável associada ao macho, obtendo o modelo 4 :

$$
Y_{i j}=m+F_{i}+\beta_{1}\left(t_{i}-\bar{t} .\right)+\beta_{2}\left(n_{i j}-\bar{n} . .\right)+e_{i j}
$$

em que $b_{2}$ é o coeficiente de regressão; $n_{i j}$, onúmero de espermatozóides; e os demais efeitos como no modelo 3.

\section{Resultados e Discussão}

\section{Indução à ovulação}

No período 21 a 31 de julho de 1998, todas as 23 fêmeas ovularam, quando induzidas por injeção intramuscular de LHRHa. No período de 5 a 18 de setembro de 1998, 19 das 23 ovularam; duas não responderam; e duas ovularam antes do tratamento com hormônio. No período de 14 a 27 de outubro de 1998, somente 13 das 23 fêmeas induzidas ovularam. Observou-se, portanto, diferença $(\mathrm{P}<0,001)$ entre épocas quanto à ovulação. Constam da Tabela 1 as médias de quadrados mínimos do peso de todos os animais e do número de óvulos, peso das desovas, índice ovócito somático e taxa de fecundação das fêmeas que ovularam.
A taxa de fecundação não-transformada variou entre 0 e $95 \%$, ficando a média geral em 56\%. A taxa média de fecundação por fêmea variou entre $15 \mathrm{e}$ $94 \%$, sendo a variância entre fêmeas diferente de zero $(\mathrm{P}<0,001)$ nos modelos 2 e 3 . No modelo 3, obteve-se efeito linear positivo $(\mathrm{P}<0,05)$ do tempo entre a verificação da ovulação e a extração dos óvulos. Pelo modelo 4 , não se verificou $(\mathrm{P}>0,10)$ efeito do número de espermatozóides, cujos valores variaram entre $1,56 \times 10^{5}$ e 1,62 x 107 , com média 1,63 x $10^{6}$; por conseguinte, este modelo foi descartado. Conclui-se, portanto, que houve variabilidade intrínseca entre fêmeas quanto à viabilidade dos óvulos. Tal variabilidade pode ter ocorrido devido à diferenças genéticas entre indivíduos.

Avaliação da técnica de fertilização artificial na produção massal de girinos

Na metodologia proposta por FALCON e CULLEY, 1995), a extração dos óvulos e a fertilização são realizadas simultaneamente. Conseqüentemente, quando os últimos óvulos são fertilizados, os primeiros já se encontram excessivamente hidratados. Segundo NAS (1974), a substância gelatinosa que envolve os ovos se torna pegajosa e aderente, dificultando o manuseio dos ovos recém-fecundados. Constatou-se que, quando a fecundação é simultânea à extração dos óvulos, há formação de grumos que dificultam a respiração dos ovos recém-fecundados e dos futuros embriões, causando mortalidade de embriões e ovos nas primeiras divisões.

Na técnica de fertilização proposta, a formação de grumos é evitada na extração dos óvulos, na diluição do esperma e na hidratação dos ovos. Os óvulos devem ser fertilizados somente após o completo esgotamento do ovário. Tal procedimento evitará a

Tabela 1 - Médias de quadrados mínimos* do peso de todas as fêmeas, do número de óvulos, do peso dos óvulos, e índice ovócito somático (IOS) das fêmeas que ovularam

Table 1 - Least square means ${ }^{\star}$ for weights of all female and eggs weights, egg numbers and somatic oocite indices of ovulating females

\begin{tabular}{lcccc}
\hline Período & $\begin{array}{c}\text { Peso das fêmeas }(\mathrm{g}) \\
\text { Female weight }(\mathrm{g})\end{array}$ & $\begin{array}{c}\text { Peso dos ovos }(\mathrm{g}) \\
\text { Egg weight }(g)\end{array}$ & $\begin{array}{c}\text { Número de óvulos }\left(\mathrm{x} 10^{4}\right)^{* *} \\
\text { Egg number }\left(x 10^{4}\right)^{* *}\end{array}$ & $\begin{array}{c}\text { IOS } \\
\text { SOI }\end{array}$ \\
\hline $\begin{array}{l}\text { Julho/1998 } \\
\text { July/1998 }\end{array}$ & $360^{\mathrm{A}}$ & $41,9^{\mathrm{B}}$ & $1,25^{\mathrm{C}}$ & $1,7^{\mathrm{A}}$ \\
$\begin{array}{l}\text { Setembro/1998 } \\
\text { September/1998 }\end{array}$ & $361^{\mathrm{A}}$ & $55,0^{\mathrm{A}}$ & $1,60^{\mathrm{A}}$ & $15,5^{\mathrm{A}}$ \\
$\begin{array}{l}\text { Outubro/1998 } \\
\text { October/1998 }\end{array}$ & $359^{\mathrm{A}}$ & $52,9^{\mathrm{AB}}$ & $1,43^{\mathrm{B}}$ & $13,8^{\mathrm{A}}$ \\
CV\% & 9,0 & 30,3 & 3,4 & 28,7 \\
\hline
\end{tabular}

* Médias, na coluna, seguidas de letras diferentes não diferem $\left(\mathrm{P} \geq^{3} 0,05\right)$ pelo teste Tukey-Kramer.

** Análise efetuada na escala logarítimica e médias retransformadas à escala original.

* Means within a column sharing the same letter do not differ $(P \geq 0,05)$ by the Tukey-Kramer test.

** Analysis on log-transformed data; means were back-transformed. 
hidratação irregular e a formação de grumos. A diluição do esperma com 100 a $200 \mathrm{~mL}$ de água facilita a mistura dos ovos com o esperma, dispensando utensílios para a homogenização, como penas ou pás. Além disso, inicia-se a hidratação dos ovos de maneira uniforme, evitando a formação de grumos. A hidratação dos ovos em 15 litros de água não permite a aderência entre os ovos, evitando a formação de grumos, e facilita a distribuição da desova nas incubadoras.

A taxa média de fecundação artificial nas 13 desovas de animais com peso médio de $360 \mathrm{~g}$ variou de 39 a $83 \%$ e o número de girinos obtidos por desova foi, em média, de 9.600. As taxas de fecundação foram semelhantes às obtidas por RIBEIRO FILHO (1998b) em acasalamentos induzidos por extrato bruto hipofisário (33 a 90\%). O número de girinos foi semelhante ao obtido por AGOSTINHO e NICTHEROY (1996) em acasalamentos induzidos por meio de hormônios sintéticos e superou os valores observados em acasalamentos naturais nos ranários comerciais, que oscilam entre 5.000 e 10.000 (LIMA e AGOSTINHO, 1992).

\section{Conclusões}

A taxa média de fertilização obtida com as modificações propostas foi superior a $60 \%$. Ressalta-se, ainda, que foram obtidas várias desovas de um mesmo animal, em curto intervalo de tempo (45 dias), aumentando a eficiência de aproveitamento do plantel de reprodutores.

As desovas induzidas em julho e nos meses subseqüentes confirmam a eficiência da técnica na sincronização da reprodução, de acordo com o fluxo de produção do ranário, independentemente da estação do ano.

Para que haja pleno aproveitamento das fêmeas, diminuindo a variabilidade intrínseca entre fêmeas, quanto à viabilidade dos óvulos, será necessário realizar estimativas da variabilidade genética entre os animais e trabalhos de seleção e melhoramento genético para esta característica.

\section{Referências Bibliográficas}

AGOSTINHO, C.A., NICTHEROY, P.E. 1996. Ensaios sobre a utilização de hormônio LHRHa na reprodução de rã-touro (Rana catesbeiana). In: REUNIÃO ANUAL DA SOCIEDADE BRASILEIRA DE ZOOTECNIA, 33, Fortaleza, Ce, 1996. Anais... Fortaleza: SBZ, 1996. p 430-431.

AGOSTINHO, C.A., NICTHEROY, P.E., PINHEIRO, D.F. BONFIM, R.M. Técnica de fertilização artificial em rã-touro
(Rana catesbeiana). In: REUNIÃO ANUAL DA SOCIEDADE BRASILEIRA DE ZOOTECNIA, 35, Botucatu, SP, 1998. Anais... Botucatu: SBZ, 1998. p.152-153.

ALONSO, M. Uso de análogos do GnRH para indução de desova e espermiação em rã touro, Rana catesbeiana Shaw, 1802. São Paulo, SP: USP, 1997. 136p. Tese (Doutorado) Universidade de São Paulo, 1991.

COSTA, C.L.S. Desenvolvimento do aparelho reprodutor $e$ fatores associados ao ciclo reprodutivo da rã-touro no sistema anfigranja. Viçosa-MG: UFV, 1991. 90p. Tese de Mestrado - Universidade Federal de Viçosa, 1991.

FALCON, G.M., CULLEY, D.D. Workshop on the reprodutive control of the bullfrog (Rana catesbeiana). In: INTERNATIONAL MEETING ON FROG RESEARCH AND TECHNOLOGY E VIII ENAR- ENCONTRO NACIONAL DE RANICULTURA, 1, Viçosa, MG. 1995. Anais... Viçosa: Abetra, 1995. p.245-246.

KRAMER, C.Y. 1957. Extension of multiple range tests to group correlated adjusted means. Biometrics, 13(1):13-18.

LIMA, S.L., AGOSTINHO, C.A. 1988. A criação de rãs. Rio de Janeiro: Editora Globo (Coleção do Agricultor). 187p.

LIMA, S.L., AGOSTINHO, C.A. 1992. A tecnologia de criação de rãs. Viçosa: UFV, Imprensa Universitária. 168p.

MARTOFF, B.S. 1953. Territoriality in the green frog, Rana clamimatans. Ecology 34(1):166-174.

NATIONAL ACADEMY OF SCIENCE - NAS. 1974. Amphibians: Guidelines for the breeding, care, and management of laboratory animals. National Research Council. Institute of Laboratory Animal Resources. Subcommittee on Amphibian Standards. 152p.

RIBEIRO FILHO, O.P., LIMA, S.L., ANDRADE, D.R., SEIXAS FILHO, J.T. 1998a. Reprodução induzida de rã-touro (Rana catesbeiana, Shaw, 1802) com o uso de extrato bruto hipofisário. R. Bras. Zootec., 27(2):216-223.

RIBEIRO FILHO, O.P., LIMA, S.L., ANDRADE, D.R., SEIXAS FILHO, J.T. 1998b. Estudo da desova de rã-touro, Rana catesbeiana, mediante indução do acasalamento. R. Bras. Zootec., 27(4):658-663.

RUGH, R.1935. Pituitary - induced sexual reactions in the anura. Biol. Bull., 68:74-81.

SAS INSTITUTE. 1985. SAS User's Guide. Cary, NC, 380p. WOYNAROVICH, E., HORVVATH, L.A. 1983. Propagação artificial de peixes de águas tropicais. Manual de extensão. Brasília-DF: FAO/CODEVASF/CNPq. 80p.

Recebido em: 13/10/99

Aceito em: 28/03/00 